NTUA-99-2001

\title{
Inflation Induced by Vacuum Energy and Graceful Exit from it.
}

\author{
E. Papantonopoulos ${ }^{a}$ and I. Pappa ${ }^{b}$ \\ National Technical University of Athens, Physics Department, Zografou \\ Campus, GR 157 80, Athens, Greece.
}

\begin{abstract}
Motivated by brane cosmology we solve the Einstein equations with a time dependent cosmological constant. Assuming that at an early epoch the vacuum energy scales as $1 / \log$, we show that the universe passes from a fast growing phase (inflation) to an expanding phase in a natural way.
\end{abstract}

${ }^{a}$ e-mail address:lpapa@central.ntua.gr

${ }^{b}$ e-mail address:gpappa@central.ntua.gr 


\section{Introduction}

The cosmological constant remains one of the biggest mysteries of our universe. Recent observations of type Ia Super Novae suggest that the expansion of the universe is accelerating now [1]. This acceleration could be explained by a positive time dependent cosmological constant with $\Omega_{\Lambda} \sim 0.7$. This suggest that the cosmological constant is almost the same order of magnitude as the present mass density of the universe.

If we accept that the cosmological constant is a time dependent quantity, to explain its present value it should be decaying with time. If we parametrize it as $\Lambda=t^{-n}$ then considerations on the age of the universe constrain the value of $\mathrm{n}$. Adopting the commonly used value of Hubble parameter $H_{0}=$ $73 \pm 10 \mathrm{kms}^{-1} \mathrm{Mpc}^{-1}$ [2], the value of $\mathrm{n}$ is fixed to $\mathrm{n}=2$. At the early stages of the cosmological evolution we expect the cosmological constant to be large but we do not know anything about its nature and how it scales with time.

Recently the idea that we are living on a D3-brane was proposed. In this brane universe scenario, the Standard Model gauge bosons as well as charged matter arise as fluctuations of the D-branes. The universe is living on a collection of coincident branes, while gravity and other universal interactions is living in the bulk space [5]. For all such theories, an essential issue concerns the cosmological evolution of our universe. In the literature, there are a lot of cosmological models associated to brane universe [6]-10]. In a class of these models, specially when there is a movement of the brane in the bulk [8]-10], there is always a time dependent cosmological constant which is induced on the brane [11]- [14].

In 14 we studied the motion of the brane universe in a type 0 string background. We calculated the energy density which is induced on the brane because of its movement in the background. What we found is that there is an inflationary phase of the brane universe which is followed by another phase in which the scale factor $\alpha$ is slowing down and the energy density is dominated by a term proportional to $\frac{1}{(\log (\alpha))^{4}}$. We attributed this behaviour to "mirage" matter which is induced on the brane because of the specific type-0 string background.

In this work we will study this effect in details. Our brane cosmological picture tells us that an effective energy density is induced which controls the cosmological evolution of the brane universe. This effective energy density plays the rôle of a time dependent cosmological constant. Motivated by this result, we will examine the effect of a time dependent cosmological constant 
to the usual Friedman-Robertson-Walker Universe. We will consider the Einstein equations with a cosmological constant $\Lambda(t)=\frac{1}{\log t}$, and concentrate on the exact solutions which exhibit inflationary behaviour and graceful exit from inflation.

What we found is that the universe at its early stages of evolution has two phases. One is of rapid expansion (inflation), with large cosmological constant and an energy density which decreases and reaches the critical energy density at the end of inflation. This phase is followed by a phase of slow expansion during which the cosmological constant remains large. The transition from one phase to the other is continuous and smooth, without having any singularity.

Our work is organized as follows. In section two, we discuss the general framework of a brane moving in the background of other branes. In section three we solve the Einstein equations with a time dependent cosmological constant, and finally in section four we discuss our results.

\section{Brane cosmology}

There are various ways to do cosmology on the brane. The more conventional way is to assume that the brane is static comparable to the bulk background, and is situated in a particular point $r_{0}$ of the radial coordinate. Then matter on the brane or in the bulk can lead to a cosmological evolution of the brane universe. Another approach is to consider a brane moving in the background of other branes. Then one can show that even if there is no matter on the brane, the brane universe undergoes a cosmological evolution as a result of its movement [8]-[15].

The simplest way to follow such a movement is to assume that the brane as it moves in the bulk does not back react with the geometry. In 11 and [14] we considered a probe brane moving on a geodesic in a generic static, spherically symmetric background [10]. The metric of a D3-brane was parametrized as

$$
d s_{10}^{2}=g_{00}(r) d t^{2}+g(r)(d \vec{x})^{2}+g_{r r}(r) d r^{2}+g_{S}(r) d \Omega_{5}
$$

In the background there is also a dilaton field $\Phi$ and a $R R$ background $C(r)=$ $C_{0 \ldots 3}(r)$ with a self-dual field strength. The dynamics on the brane will be governed by the Dirac-Born-Infeld action given by 


$$
\begin{aligned}
S= & T_{3} \int d^{4} \xi e^{-\Phi} \sqrt{-\operatorname{det}\left(\hat{G}_{\alpha \beta}+\left(2 \pi \alpha^{\prime}\right) F_{\alpha \beta}-B_{\alpha \beta}\right)} \\
& +T_{3} \int d^{4} \xi \hat{C}_{4}+\text { anomaly terms }
\end{aligned}
$$

One can show that the induced metric on the brane is [10]

$$
d \hat{s}^{2}=-d \eta^{2}+g(r(\eta))(d \vec{x})^{2}
$$

with $\eta$ the cosmic time which is defined in terms of the background fields by

$$
d \eta=\frac{\left|g_{00}\right| g^{\frac{3}{2}} e^{-\Phi}}{|C+E|} d t
$$

The induced metric on the brane, is the standard form of a flat expanding universe. For this metric we can write the effective Einstein equations on the brane,

$$
R_{\mu \nu}-\frac{1}{2} g_{\mu \nu} R=8 \pi G\left(T_{\mu \nu}\right)_{e f f}
$$

where $\left(T_{\mu \nu}\right)_{\text {eff }}$ is the effective energy momentum tensor which is induced on the brane. We have assumed that our brane is light and there is no backreaction with the bulk. We expect $\left(T_{\mu \nu}\right)_{e f f}$ to be a function of the quantities of the bulk. If we now assume the usual form of a perfect fluid for the effective energy momentum tensor, we get from (5)

$$
8 \pi G \rho+\Lambda=\frac{3}{4} g^{-2} \dot{g}^{2}
$$

We can define a $\rho_{\text {eff }}$ from the relation

$$
8 \pi G \rho+\Lambda=8 \pi G_{N} \rho_{e f f}
$$

Using equation (4) we get

$$
\dot{g}=g^{\prime}\left[\frac{\left|g_{00}\right|}{g_{r r}}-\frac{g_{00}^{2}}{g_{s} g_{r r}}\left(\frac{g^{3} g_{s} e^{-2 \Phi}+\ell^{2}}{(C+E)^{2}}\right)\right]^{\frac{1}{2}} \frac{|C+E|}{\left|g_{00}\right| g^{\frac{3}{2}} e^{-\Phi}}
$$

where prime denotes differentiation with respect to $r$. To derive an analogue of the four dimensional Friedman equations for the expanding four dimensional universe on the probe D3-brane, we define the scale factor as $g=\alpha^{2}$ and then equation (7) with the use of (6) and (8) becomes

$$
\frac{8 \pi}{3} G_{N} \rho_{e f f}=\left(\frac{\dot{\alpha}}{\alpha}\right)^{2}=\frac{(C+E)^{2} g_{S} e^{2 \Phi}-\left|g_{00}\right|\left(g_{S} g^{3}+\ell^{2} e^{2 \Phi}\right)}{4\left|g_{00}\right| g_{r r} g_{S} g^{3}}\left(\frac{g^{\prime}}{g}\right)^{2}
$$


Therefore the motion of a D3-brane on a general spherically symmetric background had induced on the brane a matter density. As it is obvious from the above relation, the specific form of the background will determine the cosmological evolution on the brane. If we go now to a particular background namely of a type 0 string, we get for $\rho_{\text {eff }}$ 14

$$
\begin{aligned}
\frac{8 \pi}{3} \rho_{e f f}= & {\left[\left(1-\frac{1}{Q \alpha^{4}} E i[\log 2 Q+4 \log \alpha]+\frac{E}{2 \alpha^{4}}\right)^{2}\right.} \\
& \left.-\frac{1}{4}\left(1-\frac{1}{2(\log 2 Q+4 \log \alpha)}\right)^{4}\right]\left(1-\frac{1}{2(\log 2 Q+4 \log \alpha)}\right)^{-4} \\
& \left(1-\frac{9}{2(\log 2 Q+4 \log \alpha)}\right)^{-1} \\
& \left(1+\frac{1}{(\log 2 Q+4 \log \alpha)^{2}} \frac{1}{\left(1-\frac{1}{2(\log 2 Q+4 \log \alpha)}\right)}\right)^{2}
\end{aligned}
$$

As we can see from the above relation, the brane universe has an inflationary phase corresponding to the constant term. As the scale factor $\alpha$ evolves, the term $\frac{1}{(\log \alpha)^{4}}$ starts to contribute to $\rho_{\text {eff }}$ having the effect of slowing down the exponential growth. Therefore on the brane we have an inflationary phase which is followed by a less rapid expansion.

The energy density induced on the brane is time dependent, because the brane moves in the bulk. This time dependent energy density, effectively acts, as a time dependent cosmological constant. One should be careful though of introducing a time dependent cosmological constant in the usual four-dimensional Einstein equations. Consider the four-dimensional Einstein equations with a time dependent cosmological constant $\Lambda$

$$
G_{\mu \nu}=8 \pi G T_{\mu \nu}-\Lambda g_{\mu \nu}
$$

where $G_{\mu \nu}=R_{\mu \nu}-R g_{\mu \nu} / 2$ is the Einstein tensor and $T_{\mu \nu}$ is the energy momentum tensor. Taking the covariant divergence of equation (11) and assuming the conservation law $\nabla^{\nu} T_{\mu \nu}$ it follows that $\Lambda=$ constant. Therefore a time dependent cosmological constant violates energy conservation in general relativity.

After the recent astrophysical observations, we know that the dominant contribution to the energy density comes from the invisible or dark matter. Then one can define an effective energy momentum tensor by

$$
\tilde{T}_{\mu \nu} \equiv T_{\mu \nu}-\frac{\Lambda}{8 \pi G} g_{\mu \nu}
$$


where $T_{\mu \nu}$ is the contribution of the normal matter and $\frac{\Lambda}{8 \pi G} g_{\mu \nu}$ is the dark matter component of the energy momentum tensor. Then these is no any compelling season why $\tilde{T}_{\mu \nu}$ should not be conserved.

A time dependent cosmological constant, except its usefulness in the standard cosmology to parametrize the unknown nature of the dark matter [3], it is a very useful concept in brane cosmology. The brane as it moves, exchanges energy with the bulk and the presence of a time dependent cosmological constant is necessary to maintain the energy conservation. An observer in our brane-universe has as a dynamical variable the scale factor $\alpha$. Therefore all the quantities in his world are expressed as functions of $\alpha$ and he writes the Einstein equations as relations (5). For this observer the energy momentum tensor $\left(T_{\mu \nu}\right)_{e f f}$ is a well behaved conserved quantity. An observer now in the bulk can write the Einstein equations as the relations (11). For him the energy momentum tensor $T_{\mu \nu}$ and the cosmological constant $\Lambda$ are functions of the radial coordinate $r$. As the brane moves along the radial coordinate, there is a constant flow of energy between the bulk and the brane. The two pictures will coincide eventually, when the brane is in large radial distance and does not feel the gravitational field of the other branes.

One can see more clearly the transfer of energy between the bulk and the brane in the case where the brane is "fat" and back-reacts with the background. Assume that the bulk is D-dimensional and contains a brane (domain wall). Demanding that the metric be continuous everywhere and that the derivatives of the metric be continuous everywhere except on the brane, one can derive, using the Israel equations, the equations [8

$$
\bar{\nabla}_{\mu} t^{\mu \nu}=-\left\{\bar{\nabla}_{\mu} K^{\mu \nu}-h^{\mu \nu} \bar{\nabla}_{\mu} K\right\}
$$

where $t_{\mu \nu}$ is the energy momentum tensor on the brane $K_{\mu \nu}$ is the extrinsic curvature and $h_{\mu \nu}$ is the induced metric on the brane. This equation shows that the energy on the brane is not conserved. Using Codacci's equation we get

$$
\bar{\nabla}_{\mu} t^{\mu \nu}=-\left\{h^{\nu \mu} T_{\mu p} n^{p}\right\}
$$

where $T_{\mu \nu}$ is the energy momentum tensor of the bulk. This equation describes conservation of energy as it flows from the bulk to the brane and vice versa. 


\section{Einstein equations with a time dependent cosmological constant}

Motivated by our brane universe picture, we will assume that at early stages of cosmological evolution, there is a time dependent effective cosmological constant, which scales as $\Lambda(t)=\frac{1}{\operatorname{logt}}$, and study its effect to the usual Friedman-Robertson-Walker universe. We will work in a homogeneous and isotropic Universe with the energy momentum tensor having the form of a perfect fluid with pressure $\mathrm{p}$ and energy density $\rho$. In accordance to equation (12) we define an effective pressure $\tilde{p}$ and an effective energy density $\tilde{\rho}$ from

$$
\begin{aligned}
\tilde{p} & \equiv p-\frac{\Lambda}{8 \pi G} \\
\tilde{\rho} & \equiv \rho-\frac{\Lambda}{8 \pi G}
\end{aligned}
$$

The field equations (11) and the energy conservation give

$$
\begin{gathered}
\dot{\alpha}^{2}=\frac{8 \pi G}{3} \rho \alpha^{2}+\frac{\Lambda}{3} \alpha^{2}-k \\
\frac{d}{d \alpha}\left(\rho \alpha^{3 \gamma}\right)=-\left(\frac{\alpha^{3 \gamma}}{8 \pi G}\right) \frac{d \Lambda}{d \alpha}
\end{gathered}
$$

In deriving equation (17) we have taken as an equation of state

$$
p=(\gamma-1) \rho
$$

with $\gamma=$ constant. We get the dust-dominated universe for $\gamma=1$ and the radiation-dominated universe for $\gamma=4 / 3$. Differentiating equation (16) and using (17) we get

$$
\ddot{\alpha}=\frac{8 \pi G}{3}\left(1-\frac{3 \gamma}{2}\right) \rho \alpha+\frac{\Lambda}{3} \alpha
$$

The above equation shows that if $\gamma>2 / 3$ a positive $\rho$ acts to decelerate the expansion, while if $\gamma<2 / 3$ the density accelerates the expansion. Combining equations (16) and (19) we get

$$
\frac{\ddot{\alpha}}{\alpha}=\left(1-\frac{3 \gamma}{2}\right)\left(\frac{\dot{\alpha}^{2}}{\alpha^{2}}+\frac{k}{\alpha^{2}}\right)+\frac{\gamma}{2} \Lambda
$$


This is the equation that governs the behavior of the scale factor in the presence of a cosmological term $\Lambda$. Introducing $H=\frac{\dot{\alpha}}{\alpha}$ we obtain from (20)

$$
\dot{H}=-\frac{3 \gamma}{2} H^{2}+\frac{\gamma}{2} \Lambda+\left(1-\frac{3 \gamma}{2}\right) \frac{k}{\alpha^{2}}
$$

We will work with spatially flat universes and in this case because $\mathrm{k}=0$ the last term of equation (21) drops off leaving a special case of Riccati's equation [1]. Defining a new variable $x$ from the relation

$$
H=\left(\frac{2}{3 \gamma}\right) \frac{\dot{x}}{x}
$$

equation (21) becomes

$$
\frac{\ddot{x}}{x}-\frac{3 \gamma^{2}}{4} \Lambda=0
$$

We take $\Lambda(t)=\frac{1}{\log t}$ and to set a time scale, we know that the inflationary period of the universe is well before the nucleosynthesis. Therefore our assumption is valid until a cutoff time $t_{c}$, the time before the nucleosynthesis starts,

$$
\Lambda=\left\{\begin{array}{c}
\frac{A}{\log t} \text { when } t<t_{c} \\
\Lambda_{c} \text { when } t>t_{c}
\end{array}\right.
$$

where $\mathrm{A}$ is a constant. This choice of $t_{c}$ is consistent with our brane world picture. We expect that this time dependent effective cosmological constant will receive various contributions from other interactions, mainly gauge interactions, at later cosmological evolution stages. Substituting $\Lambda$ of (24) in the equation (23) we get

$$
(\log t) \ddot{x}-a x=0
$$

where $a=\frac{3}{4} \gamma^{2} A$. We go again to a new variable $z$, defined by $t=\exp \left(-b z^{q}\right)$ where $b$ and q are constants. Then equation (25) becomes

$$
z^{2-q} \frac{d^{2} x}{d z^{2}}+\left((1-q) z^{1-q}+b q z\right) \frac{d x}{d z}+a b q^{2} x e^{-2 b z^{q}}=0
$$

We are looking for solutions of the above equation for various values of the parameters $q, a$, and b. To simplify our solutions we fix $q=1$. Before we discuss in detail the solutions, let us assume that we have a solution $\mathrm{x}(\mathrm{z})$ of the equation (26). Then using the transformation $t=e^{-b z}$ we get $\mathrm{x}(\mathrm{t})$ and from (22) the scale factor is

$$
\alpha(t)=[x(t)]^{2 / 3 \gamma}
$$


To solve equation (26) we first consider the case where $\mathrm{b}=1$. Then $t=e^{-z}$ and equation (26) becomes

$$
\frac{d^{2} x}{d z^{2}}+\frac{d x}{d z}+a \frac{e^{-2 z}}{z} x=0
$$

This equation cannot be solved analytically. Nevertheless for small values of $\mathrm{z}$ equation (28) can be approximated by the equation

$$
\frac{d^{2} x}{d z^{2}}+\frac{d x}{d z}+a \frac{x}{z}=0
$$

This equation can give exact solutions for various values of $a$. If $a= \pm 1$ we get the following solutions

$$
\begin{array}{ccc}
x(z)=c_{1} e^{-z} z+c_{2} e^{-z} z \Gamma(-1,-z) & \text { when } & a=1 \\
x(z)=z c_{1}+c_{2} \text { Meijer } G & \text { when } a=-1
\end{array}
$$

Where $c_{1}$ and $c_{2}$ are constants to be fixed by initial conditions, end MeijerG is the Meijer $\mathrm{G}$ function $G_{10}^{20}\left(\left.z\right|_{0,1} ^{2}\right)$. Equation (28) for large values of $z$ can be approximated by the equation

$$
\frac{d^{2} x}{d z^{2}}+\frac{d x}{d z}+a e^{-2 z} x=0
$$

This equation can be solved analytically and for $a= \pm 1$ we get two solutions

$$
\begin{gathered}
x(z)=c_{1} \cos \left(e^{-z}\right)+c_{2} \sin \left(e^{-z}\right) \quad \text { when } \quad a=1 \\
x(z)=c_{1} \cosh \left(e^{-z}\right)+i c_{2} \sinh \left(e^{-z}\right) \text { when } a=-1
\end{gathered}
$$

We will discuss first the case of $a=1$. Inserting the solutions for $a=1$ in (27) we get

$$
\begin{array}{ccc}
\alpha(t)=c_{1}^{\prime} t^{2 / 3 \gamma}(-\log t)^{2 / 3 \gamma} & \text { small } & t \\
\alpha(t)=c_{1}^{\prime}(\cos t)^{2 / 3 \gamma} & \text { large } & t
\end{array}
$$

We have absorbed the factor $2 / 3 \gamma$ into the definition of $c_{1}^{\prime}$ and have put $c_{2}=0$. This solution does not have an inflationary phase and therefore we do not discuss it any further. For $a=-1$ using (27) we get

$$
\begin{array}{ccc}
\alpha(t)=c_{1}^{\prime}(-\log t)^{2 / 3 \gamma} & \text { large } & t \\
\alpha(t)=\frac{c_{1}^{\prime}}{2}\left(e^{t}+e^{-t}\right)^{2 / 3 \gamma} & \text { small } & t
\end{array}
$$


where again we have absorbed the factor $2 / 3 \gamma$ into the definition of $c_{1}^{\prime}$ and have put $c_{2}=0$. This is an interesting cosmological solution. The universe in an early epoch has two phases: one is rapid expansion (inflation) with positive cosmological constant $\Lambda$ and a slow expansion phase also with positive cosmological constant. The crucial question is how these two phases are connected. We performed a numerical investigation of equation (28) for $a=-1$ and for a wide range of values of $z$. What we found is that the two phases are connected in a smooth way in the sense that the scale factor $\alpha(t)$ is passing from one phase to the other without having any singularity.

Another interesting question is for how long the inflation lasts. We can answer this question calculating the number of e-foldings. The number of e-foldings is given by

$$
N=\int_{t_{i}}^{t_{f}} H(t) d t
$$

where $t_{i}$ is the time when inflation starts and $t_{f}$ the time it ends. Using equation (22) and the solution for $x(z)$ from (32) we get

$$
N=\frac{2}{3 \gamma} \ln \frac{\cosh t_{f}}{\cosh t_{i}}
$$

Demanding $N>60$ we get

$$
\cosh _{f}>\cosh _{i} e^{90 \gamma}
$$

To satisfy the above equation for a very small time $t_{i}, \gamma$ should be positive and very small. This value of $\gamma$ is consistent with the "inflationary zone" $\gamma<\frac{2}{3}$ and the expectation that during inflation, the pressure $p$ is negative.

We can also find how the energy density varies during the two phases. Using (34), the equation for the energy density (17) becomes for the inflationary phase

$$
\dot{\rho}+2 \frac{e^{t}-e^{-t}}{e^{t}+e^{-t}} \rho+\frac{4}{24 \pi G \gamma^{2}} \frac{1}{\left(t(\log t)^{2}\right)}=0
$$

Note that the energy density does not depend on the initial conditions of the scale factor but only on $\gamma$, and the initial value of $\rho$. A numerical analysis of the above equation shows that as the universe expands the energy decreases. To solve the flatness problem, we expect the energy density $\rho$ at the end of inflation to reach $\rho_{c}$ which is given by

$$
\rho_{c}=\frac{3 H^{2}}{8 \pi G}
$$


Ii our case if we define $\Omega=\frac{\rho}{\rho_{c}}$ and use (16) we get for $\mathrm{k}=0$

$$
|\Omega(t)-1|=\frac{\Lambda}{3 H^{2}}
$$

Then using (22) with the solution $x(z)$ from (32) we get

$$
|\Omega(t)-1|=-\frac{(\operatorname{coth} t)^{2}}{\log t}
$$

The right hand side of this relation tends to zero for "large" times where our approximation is still valid and the inflation terminates. Therefore $\rho \longrightarrow \rho_{c}$ as expected.

For the slow expansion phase, the energy density is given by the equation

$$
\dot{\rho}+2 \frac{\rho}{t(\log t)}+\frac{4}{24 \pi G \gamma^{2}} \frac{1}{\left(t(\log t)^{2}\right)}=0
$$

We expect that during this phase the reheating starts. The vacuum energy remains positive and is increasing providing the energy for the reheating.

The second class of solutions are generated by taking $b=-1$. These solutions are similar to $b=1$ case, but we are giving them for the completeness of our discussion. In this second case the equation (26) for small $z$ can be approximated by

$$
\frac{d^{2} x}{d z^{2}}-\frac{d x}{d z}+a \frac{x}{z}=0
$$

giving the solutions

$$
\begin{gathered}
x(z)=c_{1} z+c_{2} \text { Meijer } G \quad \text { when } \quad a=1 \\
x(z)=c_{1} e^{z} z+c_{2} e^{z} z \Gamma(-1, z) \text { when } a=-1
\end{gathered}
$$

where MeijerG is the Meijer G function $G_{10}^{20}\left(-\left.z\right|_{0,1} ^{2}\right)$. For large $z$, using an equation similar to (31) we get

$$
\begin{gathered}
x(z)=c_{1} e^{-i e^{z}}-\frac{1}{2} c_{2} i e^{i e^{z}} \quad \text { when } \quad a=1 \\
x(z)=c_{1} e^{e^{z}}-\frac{1}{2} c_{2} i e^{-e^{z}} \text { when } a=-1
\end{gathered}
$$

For $a=1$ we can not get any meaningful solution because $x(z)$ from equation (45) is imaginary. For $a=-1$ the scale factor is

$$
\begin{array}{ccc}
\alpha(t)=c_{1}^{\prime}(t \log t)^{2 / 3 \gamma} & \text { small } & t \\
\alpha(t)=c_{1}^{\prime} e^{\frac{2 t}{3 \gamma}} & \text { large } & t
\end{array}
$$


Again here we have an interesting cosmological solution with two phases one of rapid expansion and the other of slow growth connected in a smooth way. Note that inflation occurs at late cosmological times.

\section{Discussion}

We have studied the Einstein equations with a time dependent cosmological constant. What we have found is that when $\Lambda(t)=\frac{1}{\log t}$ there are exact solutions of the Einstein equations which exhibit two distinct phases of the cosmological evolution. The first phase is an inflationary phase and the second is a slow expanding phase. These two phases are connected by a smooth way without any singularity. Such cosmological evolution can be realized in a brane universe scenario. In a situation where a probe brane is moving in the gravitational field which is produced by other branes, a time dependent cosmological constant will be induced on the brane, which drives the inflation and at later times terminates it.

Our model resembles the old DeSitter model. In that model the vacuum energy is constant. This constant vacuum energy can drive the universe to a flat geometry but this evolution if it is allowed to continue will eventually produce an empty universe.

In our model the vacuum energy is time dependent and has a large value comparable to its present value. Our aim was to show that the inflationary phase terminates and the scale factor follows a slow expansion in a continuous way. For this reason we cut-off the vacuum energy for some time before the nucleosynthesis. We do not know how the vacuum energy will evolve for later times and reach its present value. This is consistent with our braneworld scenario, which is the motivation of this work. We expect that the vacuum energy will receive various contributions from other interactions, mainly gauge interactions at later cosmological times.

We had studied some very basic tests of our model, like the duration of inflation and how the energy density varies with time. A more detailed phenomenological analysis is needed and problems like the reheating of the universe should be answered. Finally a more general form of the cosmological constant $\Lambda(t)=\left(\frac{1}{\log \alpha(t)}\right)^{q}$ should be investigated [19]. 


\section{Acknowledgement}

We would like to thank C. Bachas, A. Kehagias and E. Kiritsis for valuable discussions. We thank specially N. Mavromatos for his constructive remarks. Partially supported by NTUA program Archimedes.

\section{References}

[1] S. Perlmutter et al., Nature 391, 51 (1998); A. G. Riess et al., Astron. J. 116, 1009 (1998); P. M. Garnavich et al., Astrophys. J. 509, 74 (1998); S. Permutter et al., Astrophys. J. 530, 17 (2000).

[2] W. L. Freedman, Los Alamos report astro-ph/9612024 (1996).

[3] E. B. Gliner, Sov. Phys. JETP 22, 378 (1966); Y. B. Zel'dovich, Sov. Phys. Usp. 11, 381 (1968); A. D. Linde, JETP letters 19, 183 (1974); A. M. Polyakov, Sov. Phys. Ups. 25, 187 (1982); S. L. Adler, Rev. Mod. Phys. 54729 (1982).

[4] J. M. Overduin and F. I. Cooperstock, Phys. Rev. D58 043506 (1998).

[5] J.Polchinski, Dirichlet branes and Ramond-Ramond charges, Phys. Rev. Lett. 75 (1995) 4724 hep-th/9510017

[6] N.Kaloper and A.Linde, Inflation and large internal dimensions, Phys. Rev. D 59 (1999) 101303 hep-th/9811141;

N.Arkani-Hamed, S.Dimopoulos, N.Kaloper and J.March-Russell, Rapid asymmetric inflation and early cosmology in theories with submillimeter dimensions, hep-ph/9903224;

N.Arkani-Hamed, S.Dimopoulos, G.Dvali and N.Kaloper, Infinitely large new dimensions, hep-th/9907209;

N.Kaloper. Bent domain walls as brane-worlds, Phys. Rev. D60 (1999) 123506 hep-th/9905210.

P.Kanti, I.I.Kogan, K.A.Olive and M.Pospelov, Cosmological 3-Brane Solution, Phys. Lett. B468 (1999) 31 hep-ph/9909481

[7] P.Binetruy, C.Deffayet and D.Langlois, Nonconventional cosmology from a brane universe, hep-th/9905012. 
G.Dvali and S.H.H.Tye, Brane inflation, Phys. Lett. B450 (1999) 72 hep-ph/9812483;

E.E.Flanagan, S.H.H.Tye and I.Wasserman, A cosmology of the brane world, hep-ph/9909373.

H.B.Kim and H.D.Kim, Inflation and Gauge Hierarchy in RandallSundrum Compactification, hep-th/9909053

C.Csaki, M.Graesser, C.Kolda and J.Terning, Cosmology of One Extra Dimension with Localized Gravity, Phys. Lett. B462 (1999) 34 hepph/9906513

C.Csaki, M.Graesser, L.Randall and J.Terning, Cosmology of Brane Models with Radion Stabilization, hep-ph/9911406

S. Nojiri, O. Obregon and S. D. Odintsov, (Non)-Singular Brane-World Induced by Quantum Effects in D5 Dilatonic Gravity, Phys. Rev., D62 (2000) 104003, hep-th/0101003.

[8] H.A.Chamblin and H.S.Reall, Dynamic dilatonic domain walls, hepth/9903225; A.Chamblin, M.J. Perry and H.S.Reall, Non-BPS D8branes and dynamic domain walls in massive IIA supergravities, J.High Energy Phys. 09 (1999) 014 hep-th/9908047.

P.Kraus, Dynamics of Anti-de Sitter Domain Walls, JHEP 9912:011 (1999) hep-th/9910149

[9] J. E. Ellis, N. E. Mavromatos and D. V. Nanopoulos, Time Dependent Vacuum Energy Induced by D Particle Recoil, Gen. Rel. Grav. 32 (2000) 943 gr-qc/9810086

[10] A.Kehagias and E.Kiritsis, Mirage cosmology, JHEP 9911:022 (1999) hep-th/9910174

[11] E.Papantonopoulos and I.Pappa, Type 0 Brane Inflation from Mirage Cosmology, Mod. Phys. Lett. A 15 (2000) 2145 [hep-th/0001183

[12] J.Y. Kim, Dilaton-driven brane inflation in type IIB string theory, hepth/0004155

[13] J. Y. Kim, Brane inflation in tachyonic and non-tachyonic type $O B$ string theories hep-th/0009175 
[14] E.Papantonopoulos and I.Pappa, Cosmological Evolution of a Brane Universe in a Type 0 String Background Phys. Rev. D63 (2001) 103506 hep-th/0010014

[15] D. Youm, Brane Inflation in the Background of a D-Brane with NS $B$ field, hep-th/0011024; Closed Universe in Mirage Cosmology, hepth/0011290

[16] T.Regge and C.Teitelboim, Marcel Grossman Meeting on General Relativity,Trieste 1975, North Holland;

V.A. Rubakov and M.E. Shaposhnikov, Do we live inside a domain wall? Phys. Lett.,B 125 (1983) 136.

[17] N.Arkani-Hamed, S.Dimopoulos and G.Dvali, The hierarchy problem and new dimensions at a millimeter, Phys. Lett. B 429(1998) 263 hepph/9803315]; Phenomenology, astrophysics and cosmology of theories with submillimeter dimensions and TeV scale quantum gravity, Phys. Rev.D 59 (1999) 086004 hep-ph/9807344;

I.Antoniadis, N.Arkani-Hamed, S.Dimopoulos and G.Dvali, New dimensions at a millimeter to a Fermi and superstrings at a TeV, Phys. Lett. B 436 (1998) 257 hep-ph/9804398

[18] R.Sundrum, Effective field theory for a three-brane universe, Phys. Rev. D 59 (1999) 085009 hep-ph/9805471; Compactification for a three brane universe, Phys. Rev. D 59 (1999) 085010 [hep-ph/9807348];

L.Randall and R.Sundrum, Out of this world supersymmetry breaking, Nucl. Phys. B.557 (1999) 79 hep-th/9810155]; A large mass hierarchy from a small extra dimension, Phys. Rev. Lett. 83 (1999) 3370 hep$\mathrm{ph} / 9905221$.

[19] E. Papantonopoulos and I. Pappa in preparation 